\title{
Lycophytes and monilophytes in Rio Preto State Park, Minas Gerais, Brazil
}

\author{
Alexandre Salino $^{1,2}$, Nara Furtado de Oliveira Mota ${ }^{1}$ and Thaís Elias Almeida ${ }^{1}$
}

Submitted: 14 December, 2011. Accepted: 4 December, 2012

\begin{abstract}
We conducted a floristic survey of lycophytes and monilophytes in Rio Preto State Park, located in the municipality of São Gonçalo do Rio Preto, and in the surrounding areas, including the municipality of Felício dos Santos, in the state of Minas Gerais, Brazil. The study area is within the Espinhaço Mountain Range. Collections were made from June 1999 to August 2008, and the specimens were deposited in the Herbarium of the Federal University of Minas Gerais. Were identified 188 taxa (185 species, one subspecies and two varieties), distributed among 20 families and 60 genera. The most representative families were Polypodiaceae, with 35 species; Pteridaceae, with 24 species; Dryopteridaceae, with 23 species; Hymenophyllaceae, with 17 species; and Lycopodiaceae, with 13 species and one variety. The most species-rich genera were Elaphoglossum $(\mathrm{n}=19)$, Asplenium $(\mathrm{n}=12)$, Blechnum $(\mathrm{n}=10)$ and Thelypteris $(\mathrm{n}=9)$. Of the taxa recorded, nine are endangered species. Here we present a taxa checklist for the studied area, data regarding growth habit and vegetation type for each species, as well as comparative analyses between Rio Preto State Park and other areas within the Espinhaço Range and the Atlantic Forest, in terms of species composition.
\end{abstract}

Key words: Espinhaço Range, floristic, Rio Preto Basin, cerrado

\section{Introduction}

The Espinhaço Mountain Range, also known as the Serra Geral, encompasses a group of mountains separating the São Francisco River Basin from the Atlantic coast of Brazil (Abreu 1984). This mountain chain is bordered on the south by the Serra de Ouro Branco, in the state of Minas Gerais, and on the north by the Serra da Jacobina, in the state of Bahia (Harley 1995).

Until the 19th century, the Espinhaço Range was internationally renowned for its remarkable geomorphology, being an important source of precious minerals such as gold and diamonds (Gontijo 2008). Currently, although iron mining still thrives in the southern portion (Jacobi \& Carmo 2008), the main richness of the range is its plant and animal diversity (Gontijo 2008; Leite et al. 2008; Rapini 2008; Vasconcelos et al. 2008).

The mosaic of phytophysiognomies within the Espinhaço Range, composed of forest, grassland and savanna, provides a plurality of niches and contributes to high rates of endemism and species richness. In this context, the vegetation of the campos rupestres ("rupestrian grasslands") is notable. The rupestrian grasslands are characterized by open vegetation emerging generally above $900 \mathrm{~m}$ on a substrate of quartzite rocks and sandy soils (Giulietti et al.
1987). The disrupted and scattered distribution of these grasslands throughout the Espinhaço Range, because of its heterogeneity-macro-spatial (altitudinal, topographic and latitudinal) and micro-spatial (edaphic and microclimatic) - contributes to the high rates of plant species richness and endemism in this mountain chain (Rapini et al. 2008).

Among the 4000 plant species estimated to occur throughout the Espinhaço Range (Giulietti et al. 1997), 468 lycophytes and monilophytes have been identified (Salino \& Almeida 2008a). Seedless vascular plants are divided into two monophyletic groups: lycophytes and monilophytes (Pryer et al. 2004). The lycophytes encompass approximately 1360 species (Moran 2008) distributed among three families and 18 genera, and are characterized by the presence of microphylls and adaxial sporangium at the axillary position on the leaves, lateralized to the stem, with complete, distal dehiscence. The monilophyte lineage includes species with megaphylls and a distinct vascularization system, with the protoxylem confined to lobes of the xylem strand (Pryer et al. 2004); this lineage comprises 12,240 species (Moran 2008) distributed among 42 families (Smith et al. 2008; Rothfels et al. 2012).

Lycophytes and monilophytes show a wide diversity in terms of plant size and growth habits, as well as sporophyte and gametophyte structures. They are widespread, being

\footnotetext{
${ }^{1}$ Universidade Federal de Minas Gerais, Instituto de Ciências Biológicas, Departamento de Botânica, Belo Horizonte, MG, Brazil

${ }^{2}$ Author for correspondence: salinobh@gmail.com
} 
found in the most diverse habitat types and under various environmental conditions. Despite their broad distribution, they thrive and are more abundant in tropical environments with high humidity and rare episodes of water scarcity (Page 1979). The center of lycophyte and monilophyte diversity is in the tropics, especially in regions with rugged relief of moderate to high elevation and high rainfall or humidity. This scenario is commonly related to a great diversity of microhabitats and is favorable to colonization by various groups of lycophytes and monilophytes (Ponce et al. 2002).

To date, few studies have focused exclusively on the lycophyte and monilophyte flora of the state of Minas Gerais (see Salino \& Almeida 2009). Even fewer have been conducted in the Espinhaço Range (Salino \& Almeida 2008a), and there have been no studies carried out in the Planalto de Diamantina region, in the central portion of the Espinhaço Range. According to Drummond et al. (2005), the Planalto de Diamantina is a priority area for flora conservation in Minas Gerais and is biologically special due to the occurrence of species whose distribution is restricted to the site or are in unique environments within the state. Those authors point to mining activities, plant exploitation, cattle ranching and farming as the main anthropogenic pressures in the region. The primary recommendations for flora conservation are the creation of conservation units and the development of management plans (Drummond et al. 2005).

As pointed out by Salino \& Almeida (2008a), the Espinhaço Range presents a characteristic set of monilophytes and lycophytes species, with influence from several floristic elements, from the Andes to the Atlantic Forest. Several floristic surveys at various locations in the Espinhaço Range (Salino et al., personal communication) indicate that, despite being within the Brazilian savanna (cerrado) ecosystem, these mountains are strongly influenced by the Atlantic Forest, especially in the cloud forests (mata nebular and mata sempre-verde). According to Salino \& Almeida (2008a), several species that are typical to the coastal Atlantic Forest occur in this environment. The existence of these forests, associated with other formations such as grasslands and savanna and other typical cerrado forests (including the matas de galeria), favor the establishment not only of a high number of species but also of a quite unique and heterogeneous assemblage of species. These areas might harbor a community of lycophytes and monilophytes that does not approach the communities existing in other cerrado areas or in areas of the Atlantic Forest.

The aims of the present study were to generate a list of lycophyte and monilophyte species occurring in Rio Preto State Park (RPSP), located in the Planalto de Diamantina, extending the geographic distribution of some species; to perform comparative analyses between RPSP and other areas in the Espinhaço Range and the Atlantic Forest, in terms of species composition; and, ultimately, to contribute to increasing the knowledge of the flora of the Espinhaço Range as well as that of the state of Minas Gerais as a whole.

\section{Material and methods}

\section{Study site}

The study site (RPSP) is located in the municipality of São Gonçalo do Rio Preto (18 $05^{\circ}$ to $18^{\circ} 12^{\prime}$ S; $43^{\circ} 18^{\prime}$ to $43^{\circ} 34^{\prime} \mathrm{W}$ ), in the extreme northern region of the Planalto de Diamantina. The park was established in 1993 and spans an area of approximately 12,000 ha, comprising forest formations (mata ciliar, mata de galeria, cerradão and mata sempre-verde), savannas (cerrado sensu stricto) and grasslands (campo limpo, campo sujo and campo rupestre) (Ribeiro \& Walter 1998). The RPSP relief is predominantly mountainous, and the altitude ranges from $750 \mathrm{~m}$ to 1825 $\mathrm{m}$, the highest point being Pico Dois Irmãos. According to the Köppen climate classification system (Köppen 1931), the climate is type $\mathrm{Cwb}$, with an average annual temperature of $19^{\circ} \mathrm{C}$ and well-defined wet and dry seasons. The park is in the lower basin of the Rio Preto River, which comprises several natural springs and watercourses draining toward the Rio Jequitinhonha Basin. The park harbors remarkable floristic diversity (Lombardi et al., unpublished data) and plays an important role in the conservation and maintenance of the genetic diversity of rupestrian grasslands and related ecosystems (IEF 2004).

There is an ongoing project to increase the area of RPSP by approximately 20,000 ha. This will be achieved by annexing adjacent areas from the municipalities of Felício dos Santos, Diamantina, Rio Vermelho, Itambé do Mato Dentro and Couto de Magalhães de Minas (Antonio Augusto Tonhão de Almeida, manager of the RPSP, personal communication).

\section{Sampling and data analyses}

To collect data and register species, we carried out 32 five-day survey expeditions between June 1999 and August 2008. We sampled all the phytophysiognomies of RPSP, including a large fragment of Atlantic Forest (ca. 4000 ha), which actually lies outside the current boundaries of RPSP but will soon be included in the park, located in the vicinity of Felício dos Santos. The incorporation of this forest fragment, known as the Mata do Isidoro, into the park, is justifiable due to the importance of the area in the overall regional context, regarding the important role that this forest area plays in the composition of the pteridophyte flora in the Espinhaço Range, in the southeastern and southern regions of Brazil (Salino \& Almeida 2008a).

Samples were collected and prepared according to the usual techniques adopted for lycophytes and monilophytes (Silva 1984). Taxonomic identification was based on the specific literature and comparisons with material previously identified by specialists at the Herbarium of the Federal University of Minas Gerais. Voucher material was deposited at the herbarium. The material pertaining to this collection 
was thoroughly analyzed, and those specimens previously collected at the study site were recorded. The monilophyte taxonomic classification was based on Smith et al. (2008), whereas for lycophytes we adopted the classification proposed by Øllgaard (1992; 2012). The names of authorities were abbreviated following Pichi-Sermolli (1996).

Species composition in the RPSP was compared with that of 26 other localities within the Espinhaço Range and Atlantic Forest (Tab. 1) through nonmetric multidimensional scaling (NMDS) ordination analysis (Legendre \& Legendre 1998; Minchin 1987), using the Sørensen similarity index, with a maximum of 500 iterations. The adequacy of the ordination for interpretation was evaluated using stress value. We also performed a floristic similarity analysis using the Sørensen (Bray-Curtis) distance measure, considering that two areas would be similar when showing a value $>$ 0.5 (Magurran 2004; Kent \& Coker 1992). We created a dendrogram using the weighted pair-group method with arithmetic mean algorithm (Sokal \& Michener 1958). Both analyses were performed using the program PC-ORD 5.0 (McCune \& Mefford 2006).

\section{Results and discussion}

For RPSP, we recorded 188 infrageneric taxa (185 species, one subspecies and two varieties): 17 species and one variety of lycophytes, distributed among two families and five genera (Tab. 2); and 168 species, one subspecies and one variety of monilophytes, distributed among 19 families and 56 genera (Tab. 3). The most representative families were Polypodiaceae, with 35 species; Pteridaceae, with 24 species; Dryopteridaceae, with 23 species; Hymenophyllaceae, with 17 species; Lycopodiaceae, with 13 species and one variety; Aspleniaceae, with 12 species; Blechnaceae, with 11 species; and Thelypteridaceae, with 10 species. The genus with the highest number of species was Elaphoglossum $(\mathrm{n}=19)$, followed by Asplenium ( $\mathrm{n}=12)$, Blechnum $(\mathrm{n}=10)$, Thelypteris $(\mathrm{n}=9)$, Anemia $(\mathrm{n}=8)$ and Hymenophyllum $(\mathrm{n}=8)$.

Of the 188 taxa recorded in the present study, 128 (68.1\%) were identified exclusively in forest formations and 51 (27.1\%) were identified exclusively in grassland formations. Of those identified in forest formations, 52 were terrestrial, 43 were growing on rocks, 22 were epiphytic, three were climbers, and eight had more than one growth habit. Of those identified in grassland formations, 29 were terrestrial, 19 were growing on rocks, and three were epiphytic, the species Lycopodiella, Palhinhaea and Pseudolycopodiella predominating. The observed distribution of lycophyte and monilophyte taxa in various vegetation formations in the RPSP highlights the importance of forest environments, especially those occurring above 1400 $\mathrm{m}$, which contributes to colonization by several lycophyte and monilophyte groups and, more specifically, by species of exclusive or preferential epiphytic habit.

Two species occurring in the RPSP are included on the Official List of Endangered Species in Brazil (MMA 2008):
Asplenium schwackei and Dicksonia sellowiana. According to the Red List of Threatened Species of the Flora of the State of Minas Gerais (Drummond et al. 2008), nine species listed in the present study are under some level of threat. The species Asplenium schwackei, Cyathea bipinnatifida, Phlegmariurus itambensis and Pseudolycopodiella bejaminiana are classified as "critically endangered"; Paesia glandulosa, Jamesonia sellowiana and Pellaea gleichenioides are classified as "endangered"; Cyathea myriotricha and Dicksonia sellowiana are classified as "vulnerable". Only three subspontaneous alien species were registered in the RPSP: Nephrolepis brownii, Pteris vittata and Macrothelypteris torresiana. This shows the remarkable conservation status of the RPSP and highlights the importance of the area to the conservation of the lycophyte and monilophyte flora in Minas Gerais.

We recorded six species that are endemic to the Espinhaço Range (Salino \& Almeida 2008a): Anemia rutifolia, Asplenium geraense and Asplenium schwackei, previously known to occur only in the region of the city of Ouro Preto; Phlegmariurus itambensis, to date known to occur exclusively in Pico do Itambé State Park and Sempre Vivas National Park, also located within the Planalto de Diamantina region; Jamesonia sellowiana; and Pellaea gleichenioides.

According to Salino \& Almeida (2008a), the Planalto de Diamantina is, potentially, the third richest region in the Espinhaço Range, harboring 215 species of lycophytes and monilophytes. Of the species recorded in the present study, 12 were not on the previous species list of the Planalto de Diamantina, published by Salino \& Almeida (2008a): Dicksonia sellowiana, Lastreopsis amplissima, Sticherus lanosus, Hymenophyllum undulatum, Polyphlebium hymenophylloides, Trichomanes anadromum, Nephrolepis brownii, Oleandra articulata, Campyloneurum austrobrasilianum, Pecluma macedoi, Serpocaulon sehnemii and Adiantum serratodentatum. The results presented here show that the RPSP harbors 83\% of the lycophyte and monilophyte species occurring in the region. This richness is partially due to an increased sampling effort in this area, to the detriment of other areas in the Planalto de Diamantina region, as well as to the presence of important forest fragments contributing to increased richness of the lycophyte and monilophyte flora.

For a two-dimensional solution, the stress value in the NMDS analysis was 0.13 . This analysis revealed two different groups (Fig. 1): the first consisting of Atlantic Forest areas (coastal rain forests), coastal woodlands or seasonal semideciduous forests; and the second consisting of all of the areas in the Espinhaço Range, in addition to areas of dense rain forest in the hinterland, such as the Fernão Dias Environmentally Protected Area and Serra do Brigadeiro State Park, as well as areas of Ibitipoca State Park and Serra Negra State Park.

Four areas behaved as outliers in the NMDS analysis: the Serra do Cuscuzeiro (mountain range); Chapada Diamantina National Park; Itatiaia National Park; and Serra do Papagaio State Park. Chapada Diamantina National 
Table 1. Locations from which the pteridophyte flora was collected for use in the similarity analysis.

\begin{tabular}{|c|c|c|c|c|c|}
\hline \multirow{2}{*}{ Location } & \multirow{2}{*}{ State } & Area & Elevation & \multirow{2}{*}{$\mathrm{n}$ of species } & \multirow{2}{*}{ Reference } \\
\hline & & (ha) & $(\mathrm{m})$ & & \\
\hline Rio Preto State Park & MG & 12,185 & $750-1825$ & 189 & Present study \\
\hline Cairuçu Environmentally Protected Area & RJ & 33,800 & $0-1320$ & 115 & Sylvestre (1997) \\
\hline Fernão Dias Environmentally Protected Area & MG & 180,373 & $1000-2068$ & 173 & Melo \& Salino (2007) \\
\hline $\begin{array}{l}\text { Environmentally Protected Area of the greater metropolitan } \\
\text { area of Belo Horizonte-southern zone }\end{array}$ & MG & 2280 & $790-1420$ & 190 & Figueredo \& Salino (2005) \\
\hline Caratinga Biological Station & MG & 880 & $400-680$ & 102 & Melo \& Salino (2002) \\
\hline Duas Barras Ranch & MG/BA & 20,000 & $800-1000$ & 154 & Salino et al. (unpublished data) \\
\hline Ilha do Mel & PR & 2894 & $0-148$ & 114 & Salino et al. (2005) \\
\hline Maciço da Juréia & SP & 79,240 & $0-900$ & 86 & Prado \& Labiak (2001) \\
\hline Ibitipoca State Park & MG & 1923 & $1200-1784$ & 169 & Salino et al. (unpublished data) \\
\hline Itacolomi State Park & MG & 7000 & $660-1760$ & 170 & Rolim (2007) \\
\hline Jacupiranga State Park & SP & 150,000 & $10-1310$ & 207 & Salino \& Almeida (2008b) \\
\hline Rio Doce State Park & MG & 35,973 & $230-515$ & 116 & Melo \& Salino (2002) \\
\hline Serra do Brigadeiro State Park & MG & 14,984 & $1000-1985$ & 143 & Salino et al. (unpublished data) \\
\hline Serra do Intendente State Park & MG & 12,508 & $650-1500$ & 222 & Salino et al. (unpublished data) \\
\hline Serra do Papagaio State Park & MG & 22,917 & $800-2357$ & 75 & Salino et al. (unpublished data) \\
\hline Chapada Diamantina National Park & BA & 50,610 & $200-1800$ & 124 & Nonato (2005) \\
\hline Itatiaia National Park & $\mathrm{MG} / \mathrm{RJ}$ & 30,000 & $500-2789$ & 135 & Condack (2006) \\
\hline Serra de Itajaí National Park & SC & 57,475 & $150-940$ & 185 & Gasper \& Sevegnani (2011) \\
\hline Augusto Ruschi Biological Reserve & ES & 3598 & $800-1100$ & 126 & Aquije \& Santos (2007) \\
\hline Rio das Pedras Reserve & RJ & 1260 & 20-1050 & 114 & Mynssen \& Windisch (2004) \\
\hline Santuário do Caraça (privately owned) Nature Reserve & MG & 10,187 & $750-2072$ & 234 & Viveros (2010) \\
\hline Serra Bonita (privately owned) Nature Reserve & $\mathrm{BA}$ & 2000 & $300-1080$ & 182 & Matos et al. (2010) \\
\hline Serra do Cuscuzeiro & SP & -- & $800-1050$ & 113 & Salino (1996) \\
\hline Serra do Mar Paranaense & PR & 18,000 & $0-1889$ & 166 & Paciência (2008) \\
\hline Serra Negra & MG & 10,000 & $900-1698$ & 203 & Souza et al. (in press) \\
\hline
\end{tabular}

MG - Minas Gerais; RJ - Rio de Janeiro; BA - Bahia; PR - Paraná; SP - São Paulo; SC - Santa Catarina; ES - Espírito Santo.

Table 2. List of lycophyte species recorded in Rio Preto State Park, São Gonçalo do Rio Preto, Brazil.

\begin{tabular}{|c|c|c|c|}
\hline $\begin{array}{l}\text { FAMILY } \\
\text { Species }\end{array}$ & Growth habit & Environment & Voucher \\
\hline \multicolumn{4}{|l|}{ LYCOPODIACEAE } \\
\hline Lycopodiella alopecuroides var. integerrima (Spring) B. Øllg. \& P.G. Windisch & Terrestrial & Field & S 8027 \\
\hline L. geometra B. Øllg. \& P.G. Windisch & Terrestrial & Field & S 9347 \\
\hline Palhinhaea camporum (B. Øllg. \& P.G. Windisch) Holub & Terrestrial & Field & S 5197 \\
\hline P. cernua (L.) Vasc. \& Franco & Terrestrial & Field & S 9357 \\
\hline Phlegmariurus biformis (Hook.) B.Øllg. & Terrestrial & Field & Mt 49 \\
\hline P. flexibilis (Fée) B.Øllg. & Epiphytic & Forest & A 1469 \\
\hline P. intermedius (Trevisan) B.Øllg. & Terrestrial & Field & S 9324 \\
\hline P. itambensis (B.Øllg. \& P.G. Windisch) B.Øllg. & Terrestrial & Field & S 9356 \\
\hline P. mollicomus (Spring) B.Øllg & Epiphytic & Forest & A 1473 \\
\hline P. pungentifolius (Silveira) B.Øllg. & Terrestrial & Field & S 9324 \\
\hline P. reflexus (Lam.) B.Øllg. & Rupicolous & Forest & Mt 32 \\
\hline Pseudolycopodiella benjaminiana (P.G. Windisch) B.Øllg. & Terrestrial & Field & A 792 \\
\hline P. carnosa (Silveira) Holub & Terrestrial & Field & S 9332 \\
\hline P. caroliniana (L.) Holub & Terrestrial & Field & S 9329 \\
\hline \multicolumn{4}{|l|}{ SELAGINELLACEAE } \\
\hline Selaginella flexuosa Spring & Terrestrial & Forest & S 8029 \\
\hline S. marginata (Willd.) Spring & Terrestrial & Field & S 9346 \\
\hline S. tenuissima Fée & Rupicolous & Forest & A 795 \\
\hline S. vestiens Baker & Terrestrial & Forest & S 5213 \\
\hline
\end{tabular}

Collectors: A - T.E. Almeida; Mt - N.F.O. Mota; S - A. Salino. 
Table 3. List of monilophyte species recorded in Rio Preto State Park, São Gonçalo do Rio Preto, Brazil.

\begin{tabular}{|c|c|c|c|}
\hline $\begin{array}{l}\text { FAMILY } \\
\text { Species }\end{array}$ & Growth habit & Environment & Voucher \\
\hline \multicolumn{4}{|l|}{ ANEMIACEAE } \\
\hline Anemia elegans (Gardner) C.Presl & Rupicolous & Field & S 9308 \\
\hline A. ferruginea var. ahenobarba (Christ) Mickel & Terrestrial & Savanna & S 4751 \\
\hline A. imbricata J.W.Sturm & Terrestrial & Savanna & S 4856 \\
\hline A. lanuginosa Bong. ex J.W.Sturm & Rupicolous & Field & S 5217 \\
\hline A. oblongifolia (Cav.) Sw. & Terrestrial & Field & S 5219 \\
\hline A. raddiana Link & Terrestrial & Field & A 1481 \\
\hline A. rutifolia Mart. & Rupicolous & Field & S 5222 \\
\hline A. villosa Humb. \& Bonpl ex Willd. & Rupicolous & Forest & S 5198 \\
\hline \multicolumn{4}{|l|}{ ASPLENIACEAE } \\
\hline Asplenium auriculatum $\mathrm{Sw}$. & Rupicolous & Forest & S 9931 \\
\hline A. auritum $\mathrm{Sw}$. & Epiphytic & Forest & S 9311 \\
\hline A. cirrhatum Rich. ex Willd. & Terrestrial & Forest & S 9953 \\
\hline A. feei Kunze ex Fée & Rupicolous & Forest & S 9954 \\
\hline A. geraense (C.Chr.) Sylvestre & Epiphytic & Field & S 9345 \\
\hline A. harpeodes Kunze & Terrestrial & Forest & A 1462 \\
\hline A. incurvatum Fée & Epiphytic/Rupicolous/Terrestrial & Forest & Mt 20 \\
\hline A. pediculariifolium A.St.-Hil. & Rupicolous & Forest & S 9888 \\
\hline A. praemorsum Sw. & Epiphytic/Rupicolous & Forest & S 9360 \\
\hline A. schwackei Christ & Terrestrial & Forest & S 9913 \\
\hline A. serra Langsd. \& Fisch. & Terrestrial & Forest & S 9882 \\
\hline A. wacketii Rosenst. & Terrestrial & Forest & S 9910 \\
\hline \multicolumn{4}{|l|}{ BLECHNACEAE } \\
\hline Blechnum acutum (Desv.) Mett. & Climber & Forest & A 794 \\
\hline B. asplenioides Sw. & Rupicolous & Forest & S 5192 \\
\hline B. brasiliense Desv. & Terrestrial & Forest & A 773 \\
\hline B. cordatum (Desv.) Hieron. & Terrestrial & Field & S 9315 \\
\hline B. glaziovii Christ & Rupicolous/Terrestrial & Forest & Mt 50 \\
\hline B. occidentale L. & Terrestrial & Field & S 7997 \\
\hline B. polypodioides Raddi & Rupicolous/Terrestrial & Forest & S 5191 \\
\hline B. proliferum Rosenst. & Terrestrial & Forest & A 790 \\
\hline B. schomburgkii (Klotzsch) C.Chr. & Rupicolous & Field & S 9315 \\
\hline B. serrulatum Rich. & Terrestrial & Forest & S 5181 \\
\hline Salpichlaena volubilis (Kaulf.) J.Sm. & Climber & Forest & L 4576 \\
\hline \multicolumn{4}{|l|}{ CYATHEACEAE } \\
\hline Alsophila capensis subsp. polypodioides (Sw.) D.S.Conant & Terrestrial & Forest & A 1467 \\
\hline Cyathea bipinnatifida (Baker) Domin & Terrestrial & Forest & S 9955 \\
\hline C. corcovadensis (Raddi) Domin & Terrestrial & Forest & S 9314 \\
\hline C. delgadii Sternb. & Terrestrial & Forest & S 5173 \\
\hline C. myriotricha (Baker) R.C.Moran \& J.Prado & Rupicolous & Forest & S 5800 \\
\hline C. phalerata Mart. & Terrestrial & Forest & Ml 07 \\
\hline C. uleana (A.Samp.) Lehnert & Terrestrial & Forest & S 9950 \\
\hline C. villosa Willd. & Terrestrial & Forest & S 5792 \\
\hline \multicolumn{4}{|l|}{ DENNSTAEDTIACEAE } \\
\hline Histiopteris incisa (Thunb.) J.Sm. & Terrestrial & Field & S 9321 \\
\hline Paesia glandulosa (Sw.) Kuhn & Terrestrial & Forest & S 9337 \\
\hline Pteridium arachnoideum (Kaulf.) Maxon & Terrestrial & Savanna & S 9319 \\
\hline \multicolumn{4}{|l|}{ DICKSONIACEAE } \\
\hline Dicksonia sellowiana Hook. & Terrestrial & Forest & A 1468 \\
\hline Lophosoria quadripinnata (J.F.Gmel.) C.Chr. & Terrestrial & Forest & A 781 \\
\hline
\end{tabular}


Table 3. Continuation.

\begin{tabular}{|c|c|c|c|}
\hline $\begin{array}{l}\text { FAMILY } \\
\text { Species }\end{array}$ & Growth habit & Environment & Voucher \\
\hline \multicolumn{4}{|l|}{ DRYOPTERIDACEAE } \\
\hline Arachniodes denticulata (Sw.) Ching & Rupicolous & Field & Mt 27 \\
\hline Elaphoglossum burchellii (Baker) C.Chr. & Terrestrial & Forest & A 779 \\
\hline E. decoratum (Kunze) T.Moore & Rupicolous & Forest & S 9934 \\
\hline E. gardnerianum (Kunze ex Fée) T.Moore & Rupicolous & Forest & S 9894 \\
\hline E. gayanum (Fée) T.Moore & Rupicolous & Field & S 9330 \\
\hline E. glabellum J.Sm. & Rupicolous & Forest & S 5809 \\
\hline E. hybridum (Bory) Brack. & Rupicolous & Forest & A 1281 \\
\hline E. hymenodiastrum (Fée) Brade & Terrestrial & Forest & S 9339 \\
\hline E. iguapense Brade & Rupicolous & Forest & S 9998 \\
\hline E. langsdorffii (Hook. \& Grev.) T.Moore & Terrestrial & Forest & S 9353 \\
\hline E. lingua (C. Presl) Brack. & Rupicolous & Forest & S 9923 \\
\hline E. luridum (Fée) Christ & Rupicolous & Forest & S 9942 \\
\hline E. macahense (Fée) Rosenst. & Terrestrial & Forest & S 9352 \\
\hline E. nigrescens (Hook.) T.Moore & Epiphytic & Forest & S 9994 \\
\hline E. pachydermum (Fée) T.Moore & Terrestrial & Forest & S 9317 \\
\hline E. paulistanum Rosenst. & Rupicolous & Forest & S 9891 \\
\hline E. scalpellum (Mart.) T.Moore & Rupicolous & Field & S 9325 \\
\hline E. strictum (Raddi) T.Moore & Rupicolous & Forest & S 9895 \\
\hline E. tectum (Willd.) T.Moore & Rupicolous & Forest & S 9320 \\
\hline E. vagans (Mett.) Hieron. & Terrestrial & Field & S 9358 \\
\hline Lastreopsis amplissima (C.Presl) Tindale & Terrestrial & Forest & A 1284 \\
\hline Polybotrya speciosa Schott & Terrestrial & Forest & S 9940 \\
\hline Rumohra adiantiformis (G.Forst) Ching & Terrestrial & Field/Forest & S 9340 \\
\hline \multicolumn{4}{|l|}{ GLEICHENIACEAE } \\
\hline Dicranopteris flexuosa (Schrad.) Underw. & Rupicolous/Terrestrial & Forest & S 4737 \\
\hline D. rufinervis (Mart.) Ching & Terrestrial & Forest & S 9350 \\
\hline Sticherus lanosus (Christ) J. Gonzales & Terrestrial & Forest & S 9912 \\
\hline S. lanuginosus (Fée) Nakai & Terrestrial & Forest & S 4736 \\
\hline \multicolumn{4}{|l|}{ HYMENOPHYLLACEAE } \\
\hline Abrodictyum rigidum (Sw.) Ebihara \& Dubuisson & Terrestrial & Forest & S 5195 \\
\hline Hymenophyllum asplenioides Sw. & Rupicolous & Forest & A 1288 \\
\hline H. caudiculatum Mart. & Epiphytic/Rupicolous & Forest & A 1465 \\
\hline H. elegans Spreng & Rupicolous & Field & S 9334 \\
\hline H. fragile (Hedw.) C.V. Morton & Rupicolous & Forest & A 797 \\
\hline H. hirsutum (L.) Sw. & Rupicolous & Forest & A 799 \\
\hline H. plumosum Kaulf. & Rupicolous & Forest & A 1458 \\
\hline H. polyanthos (Sw.) Sw. & Epiphytic/Rupicolous & Field/Forest & S 9326 \\
\hline H. undulatum $\mathrm{Sw}$. & Rupicolous & Field & S 9327 \\
\hline Polyphlebium angustatum (Carmich.) Ebihara \& Dubuisson & Rupicolous & Field/Forest & Mt 28 \\
\hline P. diaphanum (Kunth) Ebihara \& Dubuisson & Rupicolous & Forest & S 9962 \\
\hline P. hymenophylloides (Bosch.) Ebihara \& Dubuisson & Rupicolous & Forest & A 1466 \\
\hline Trichomanes anadromum Rosenst. & Epiphytic & Forest & A 780 \\
\hline T. cristatum Kaulf. & Terrestrial & Forest & S 8026 \\
\hline T. pilosum Raddi & Rupicolous & Forest & S 9349 \\
\hline T. pinnatum Hedw. & Terrestrial & Forest & S 5193 \\
\hline T. polypodioides $\mathrm{L}$. & Epiphytic & Forest & A 777 \\
\hline \multicolumn{4}{|l|}{ LINDSAEACEAE } \\
\hline Lindsaea arcuata Kunze & Terrestrial & Forest & S 9937 \\
\hline L. lancea (L.) Bedd. & Terrestrial & Forest & S 5184 \\
\hline L. quadrangularis Raddi & Terrestrial & Forest & S 5186 \\
\hline L. stricta (Sw.) Dryand. & Rupicolous & Forest & S 9363 \\
\hline
\end{tabular}


Table 3. Continuation.

\begin{tabular}{|c|c|c|c|}
\hline $\begin{array}{l}\text { FAMILY } \\
\text { Species }\end{array}$ & Growth habit & Environment & Voucher \\
\hline \multicolumn{4}{|l|}{ LOMARIOPSIDACEAE } \\
\hline Nephrolepis brownii (Desv.) Hovencamp. \& Miyam. & Terrestrial & Savanna & A s.n. (BHCB 110703) \\
\hline N. cordifolia (L.) C. Presl & Terrestrial & Forest & S 9966 \\
\hline N. pectinata (Willd.) Schott & Terrestrial & Forest & A 791 \\
\hline \multicolumn{4}{|l|}{ LYGODIACEAE } \\
\hline Lygodium volubile Sw. & Climber & Forest & Mt 33 \\
\hline \multicolumn{4}{|l|}{ OLEANDRACEAE } \\
\hline Oleandra articulata (Sw.) C. Presl & Rupicolous & Forest & Mt 35 \\
\hline O. baetae Damazio & Rupicolous & Forest & A 802 \\
\hline \multicolumn{4}{|l|}{ OPHIOGLOSSACEAE } \\
\hline Ophioglossum palmatum $\mathrm{L}$. & Rupicolous & Field & V 1083 \\
\hline \multicolumn{4}{|l|}{ OSMUNDACEAE } \\
\hline Osmunda regalis $\mathrm{L}$. & Terrestrial & Field & Mt 37 \\
\hline \multicolumn{4}{|l|}{ POLYPODIACEAE } \\
\hline Alansmia reclinata (Brack.) Moguel \& M. Kessler & Rupicolous & Forest & A 1287 \\
\hline Campyloneurum austrobrasilianum (Alston) de la Sota & Epiphytic & Forest & Mt 39 \\
\hline C. centrobrasilianum Lellinger & Epiphytic & Field & S 9312 \\
\hline C. nitidum (Kaulf.) C. Presl & Rupicolous & Forest & S 9318 \\
\hline Ceradenia spixiana (M. Martens ex Mett.) L.E. Bishop & Rupicolous & Forest & A 1459 \\
\hline Cochlidium punctatum (Raddi) L.E. Bishop & Rupicolous & Field & S 9336 \\
\hline C. serrulatum (Sw.) L.E. Bishop & Rupicolous & Field & S 9335 \\
\hline $\begin{array}{l}\text { Lellingeria apiculata (Kunze ex Klotzsch) } \\
\text { A.R. Sm. \& R.C. Moran }\end{array}$ & Epiphytic & Field & S 9313 \\
\hline L. brevistipes (Mett. ex Kuhn) A.R. Sm. \& R.C. Moran & Epiphytic & Forest & Mt 25 \\
\hline Leucotrichum schenckii (Hieron.) Labiak & Epiphytic & Forest & A 796 \\
\hline Melpomene melanosticta (Kunze) A.R. Sm. \& R.C. Moran & Epiphytic & Forest & S 9971 \\
\hline $\begin{array}{l}\text { M. pilosissima (M. Martens \& Galeotti) } \\
\text { A.R. Sm. \& R.C. Moran }\end{array}$ & Epiphytic & Forest & S 9892 \\
\hline M. xiphopteroides (Liebm.) A.R. Sm. \& R.C. Moran & Epiphytic & Forest & A 1276 \\
\hline Microgramma squamulosa (Kaulf.) de la Sota & Epiphytic & Forest & S 9341 \\
\hline Moranopteris gradata (Baker) R.Y.Hirai \& J.Prado & Epiphytic & Forest & S 9974 \\
\hline Niphidium crassifolium (L.) Lellinger & Epiphytic & Forest & S 9351 \\
\hline N. rufosquamatum Lellinger & Rupicolous & Forest & S 9322 \\
\hline Pecluma macedoi (Brade) M. Kessler \& A.R. Sm. & Rupicolous & Forest & S 9900 \\
\hline P. pectinatiformis (Lindm.) M.G. Price & Terrestrial & Field & S 9310 \\
\hline P. pilosa (A.M. Evans) M. Kessler \& A.R. Sm. & Rupicolous & Forest & S 9990 \\
\hline P. recurvata (Kaulf.) M.G. Price & Rupicolous & Forest & S 9988 \\
\hline P. robusta (Fée) M. Kessler \& A.R. Sm. & Terrestrial & Forest & A 1460 \\
\hline Phlebodium pseudoaureum (Cav.) Lellinger & Rupicolous & Forest & S 5202 \\
\hline Pleopeltis astrolepis (Liebm.) E. Fourn. & Epiphytic & Forest & S 9919 \\
\hline P. hirsutissima (Raddi) de la Sota & Rupicolous & Forest & S 5208 \\
\hline P. macrocarpa (Willd.) Kaulf. & Epiphytic & Forest & S 9328 \\
\hline P. minarum (Weath.) Salino & Rupicolous & Field & S 9331 \\
\hline Serpocaulon catharinae (Langsd. \& Fisch.) A.R. Sm. & Epiphytic & Forest & S 9342 \\
\hline S. fraxinifolium (Jacq.) A.R. Sm. & Epiphytic & Forest & A 793 \\
\hline S. latipes (Langsd. \& Fisch.) A.R. Sm. & Terrestrial & Forest & S 5174 \\
\hline
\end{tabular}


Table 3. Continuation.

\begin{tabular}{|c|c|c|c|}
\hline $\begin{array}{l}\text { FAMILY } \\
\text { Species }\end{array}$ & Growth habit & Environment & Voucher \\
\hline S. mexiae (Copel.) A.R. Sm. & Rupicolous & Field & A 800 \\
\hline S. sehnemii (Pic.Serm.) Labiak \& J. Prado & Rupicolous/Terrestrial & Forest & Mt 878 \\
\hline S. triseriale (Sw.) A.R. Sm. & Terrestrial & Field & S 9348 \\
\hline S. vacillans (Link) A.R.Sm. & Terrestrial & Forest & S 9361 \\
\hline Terpsichore chrysleri (Copel.) A.R. Sm. & Epiphytic & Forest & S 9925 \\
\hline \multicolumn{4}{|l|}{ PTERIDACEAE } \\
\hline Adiantopsis flexuosa (Kunze) Link-Pérez \& Hickey & Rupicolous & Forest & S 5223 \\
\hline A. regularis (Kunze) T.Moore & Terrestrial & Forest & S 9911 \\
\hline Adiantum gracile Fée & Terrestrial & Forest & S 5803 \\
\hline A. lorentzii Hieron. & Rupicolous & Forest & A 1461 \\
\hline A. serratodentatum Willd. & Terrestrial & Savanna & S 5797 \\
\hline A. sinuosum Gardner & Terrestrial & Field & S 9307 \\
\hline A. tetraphyllum Willd. & Terrestrial & Savanna & S 4857 \\
\hline Cheilanthes bradei Prado \& A.R. Sm. & Rupicolous & Field & S 9354 \\
\hline C. goyazensis (Taub.) Domin & Rupicolous & Forest & S 5199 \\
\hline Doryopteris collina (Raddi) J.Sm. & Rupicolous & Field & S 5218 \\
\hline D. lomariacea Kaulf. & Terrestrial & Field & S 8028 \\
\hline D. ornithopus (Mett.) J.Sm. & Terrestrial & Forest & S 5804 \\
\hline Jamesonia myriophylla (Sw.) Christenh. & Rupicolous & Field & S 9355 \\
\hline J. sellowiana (Kuhn) Christenh. & Rupicolous & Field & S 9333 \\
\hline Pellaea crenata R.M. Tryon & Terrestrial & Field & N s.n. (BHCB 48580) \\
\hline P. gleichenioides (Gardner) Christ & Terrestrial & Field & S 9344 \\
\hline P. pinnata (Kaulf.) Prantl & Rupicolous & Field & S 5215 \\
\hline Pityrogramma calomelanos (L.) Link & Terrestrial & Field & S 5221 \\
\hline P. trifoliata (L.) R.M. Tryon & Terrestrial & Forest & Mt 31 \\
\hline Pteris decurrens $\mathrm{C}$. Presl & Terrestrial & Forest & Mt 22 \\
\hline P. vittata $\mathrm{L}$. & Rupicolous & Anthropogenic area & A 1470 \\
\hline Radiovittaria stipitata (Kunze) E.H. Crane & Rupicolous & Forest & S 9924 \\
\hline Vittaria graminifolia Kaulf. & Epiphytic & Forest & Mt 23 \\
\hline V. lineata (L.) Sm. & Epiphytic & Forest & Mt 9 \\
\hline \multicolumn{4}{|l|}{ SCHIZAEACEAE } \\
\hline Schizaea elegans (Vahl) Sw. & Terrestrial & Forest & S 5188 \\
\hline \multicolumn{4}{|l|}{ THELYPTERIDACEAE } \\
\hline Macrothelypteris torresiana (Gaudich.) Ching & Terrestrial & Field & A 1474 \\
\hline Thelypteris amambayensis (Christ) Ponce & Terrestrial & Forest & Mt 42 \\
\hline T. conspersa (Schrad.) A.R. Sm. & Terrestrial & Forest & Mt 38 \\
\hline T. dentata (Forssk.) E.P.St. John & Terrestrial & Forest & A 1453 \\
\hline T. glaziovii (Christ) C.F. Reed & Terrestrial & Forest & Mt 19 \\
\hline T. hispidula (Decne.) C.F. Reed & Rupicolous & Forest & A 1280 \\
\hline T. longifolia (Desv.) R.M. Tryon & Rupicolous & Forest & S 5203 \\
\hline T. opposita (Vahl) Ching & Terrestrial & Field & Mt 34 \\
\hline T. rivularioides (Fée) Abbiatti & Terrestrial & Field & S 9362 \\
\hline T. salzmannii (Fée) C.V. Morton & Terrestrial & Forest & S 9359 \\
\hline
\end{tabular}

Collectors: A - T.E. Almeida; L - J.A. Lombardi; Ml - L.C.N. Melo; Mt - N.F.O. Mota; N - M.M. Nogueira; S - A. Salino; V - P.L. Viana. BHCB - Herbarium of the Federal University of Minas Gerais. 
Table 4. Sørensen similarity index values between areas.

\begin{tabular}{|c|c|c|c|c|c|c|c|c|c|c|c|c|c|c|c|c|c|c|c|c|c|c|c|c|c|}
\hline & A & B & C & D & E & $\mathrm{F}$ & G & $\mathrm{H}$ & I & $\mathrm{J}$ & K & $\mathrm{L}$ & $\mathbf{M}$ & $\mathbf{N}$ & O & $\mathbf{P}$ & Q & $\mathbf{R}$ & $S$ & $\mathrm{~T}$ & $\mathrm{U}$ & V & W & $\mathrm{X}$ & $\mathbf{Y}$ \\
\hline A & 1 & & & & & & & & & & & & & & & & & & & & & & & & \\
\hline B & 0.646 & 1 & & & & & & & & & & & & & & & & & & & & & & & \\
\hline C & 0.627 & 0.62 & 1 & & & & & & & & & & & & & & & & & & & & & & \\
\hline D & 0.502 & 0.409 & 0.462 & 1 & & & & & & & & & & & & & & & & & & & & & \\
\hline E & 0.686 & 0.732 & 0.595 & 0.424 & 1 & & & & & & & & & & & & & & & & & & & & \\
\hline F & 0.579 & 0.646 & 0.601 & 0.386 & 0.61 & 1 & & & & & & & & & & & & & & & & & & & \\
\hline G & 0.491 & 0.53 & 0.554 & 0.353 & 0.579 & 0.495 & 1 & & & & & & & & & & & & & & & & & & \\
\hline H & 0.454 & 0.448 & 0.518 & 0.343 & 0.487 & 0.557 & 0.44 & 1 & & & & & & & & & & & & & & & & & \\
\hline I & 0.272 & 0.263 & 0.3 & 0.287 & 0.294 & 0.376 & 0.334 & 0.278 & 1 & & & & & & & & & & & & & & & & \\
\hline $\mathbf{J}$ & 0.449 & 0.423 & 0.391 & 0.309 & 0.473 & 0.438 & 0.399 & 0.391 & 0.386 & 1 & & & & & & & & & & & & & & & \\
\hline K & 0.318 & 0.292 & 0.407 & 0.329 & 0.334 & 0.393 & 0.526 & 0.415 & 0.302 & 0.307 & 1 & & & & & & & & & & & & & & \\
\hline L & 0.167 & 0.281 & 0.293 & 0.176 & 0.269 & 0.304 & 0.407 & 0.218 & 0.365 & 0.262 & 0.4 & 1 & & & & & & & & & & & & & \\
\hline M & 0.294 & 0.282 & 0.301 & 0.279 & 0.313 & 0.344 & 0.301 & 0.257 & 0.431 & 0.364 & 0.284 & 0.347 & 1 & & & & & & & & & & & & \\
\hline $\mathrm{N}$ & 0.592 & 0.598 & 0.611 & 0.397 & 0.568 & 0.61 & 0.427 & 0.504 & 0.27 & 0.365 & 0.304 & 0.18 & 0.227 & 1 & & & & & & & & & & & \\
\hline O & 0.335 & 0.365 & 0.332 & 0.327 & 0.332 & 0.393 & 0.241 & 0.472 & 0.143 & 0.279 & 0.183 & 0.061 & 0.135 & 0.424 & 1 & & & & & & & & & & \\
\hline $\mathbf{P}$ & 0.263 & 0.248 & 0.269 & 0.276 & 0.261 & 0.29 & 0.27 & 0.198 & 0.389 & 0.326 & 0.229 & 0.284 & 0.508 & 0.236 & 0.119 & 1 & & & & & & & & & \\
\hline Q & 0.311 & 0.329 & 0.337 & 0.269 & 0.31 & 0.352 & 0.21 & 0.412 & 0.144 & 0.183 & 0.154 & 0.081 & 0.145 & 0.361 & 0.452 & 0.1 & 1 & & & & & & & & \\
\hline R & 0.345 & 0.41 & 0.397 & 0.254 & 0.426 & 0.476 & 0.46 & 0.408 & 0.458 & 0.496 & 0.397 & 0.403 & 0.531 & 0.337 & 0.183 & 0.386 & 0.143 & 1 & & & & & & & \\
\hline S & 0.202 & 0.255 & 0.283 & 0.195 & 0.242 & 0.281 & 0.366 & 0.196 & 0.385 & 0.244 & 0.372 & 0.709 & 0.377 & 0.181 & 0.058 & 0.28 & 0.055 & 0.403 & 1 & & & & & & \\
\hline $\mathrm{T}$ & 0.404 & 0.398 & 0.502 & 0.299 & 0.466 & 0.545 & 0.425 & 0.572 & 0.331 & 0.431 & 0.4 & 0.266 & 0.283 & 0.468 & 0.341 & 0.184 & 0.339 & 0.46 & 0.255 & 1 & & & & & \\
\hline $\mathrm{U}$ & 0.411 & 0.458 & 0.458 & 0.342 & 0.475 & 0.516 & 0.44 & 0.458 & 0.44 & 0.488 & 0.396 & 0.345 & 0.448 & 0.411 & 0.307 & 0.421 & 0.23 & 0.65 & 0.333 & 0.48 & 1 & & & & \\
\hline V & 0.296 & 0.338 & 0.319 & 0.273 & 0.383 & 0.387 & 0.352 & 0.304 & 0.406 & 0.406 & 0.287 & 0.32 & 0.35 & 0.31 & 0.221 & 0.353 & 0.203 & 0.443 & 0.257 & 0.343 & 0.403 & 1 & & & \\
\hline W & 0.337 & 0.387 & 0.352 & 0.256 & 0.414 & 0.477 & 0.399 & 0.34 & 0.415 & 0.553 & 0.322 & 0.419 & 0.36 & 0.339 & 0.205 & 0.303 & 0.126 & 0.516 & 0.355 & 0.385 & 0.499 & 0.413 & 1 & & \\
\hline X & 0.21 & 0.22 & 0.275 & 0.256 & 0.249 & 0.331 & 0.376 & 0.246 & 0.561 & 0.333 & 0.341 & 0.402 & 0.355 & 0.232 & 0.12 & 0.328 & 0.136 & 0.436 & 0.411 & 0.363 & 0.377 & 0.339 & 0.42 & 1 & \\
\hline Y & 0.41 & 0.445 & 0.401 & 0.322 & 0.48 & 0.504 & 0.387 & 0.417 & 0.418 & 0.455 & 0.283 & 0.298 & 0.489 & 0.437 & 0.302 & 0.385 & 0.21 & 0.62 & 0.288 & 0.418 & 0.645 & 0.403 & 0.467 & 0.361 & 1 \\
\hline
\end{tabular}

A - Rio Preto State Park; B - Santuário do Caraça (privately owned) Nature Reserve; C - Itacolomi State Park; D - Chapada Diamantina National Park; E - Intendente State Park; F - Serra Negra; G - Environmentally Protected Area of the Greater metropolitan area of Belo Horizonte-southern zone; H - Fernão Dias Environmentally Protected Area; I - Cairuçu Environmentally Protected Area; J - Duas Barras Ranch; K - Serra do Cuscuzeiro; L - Caratinga Biological Station; M - Ilha do Mel; N - Ibitipoca State Park; O - Itatiaia National Park; P - Maciço da Juréia; Q - Serra do Papagaio State Park; R - Jacupiranga State Park; S - Rio Doce State Park; T - Serra do Brigadeiro State Park; U - Serra de Itajaí National Park; W - Serra Bonita (privately owned) Nature Reserve; X - Rio das Pedras Reserve.

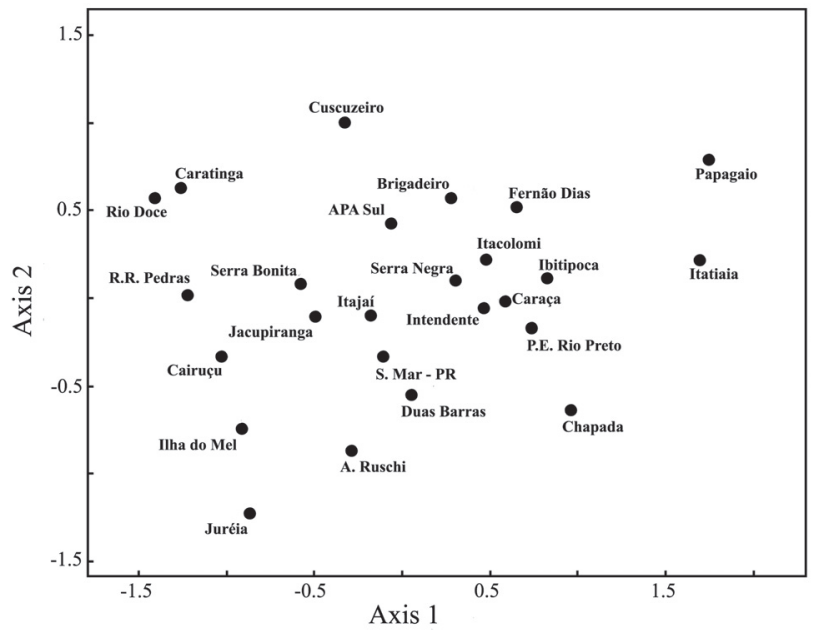

Figure 1. Nonmetric multidimensional scaling ordination analysis comparing 25 areas in terms of lycophyte and monilophyte communities, using the Sørensen (Bray-Curtis) distance measure. P.E. Rio Preto - Rio Preto State Park; Cairuçu - Cairuçu Environmentally Protected Area; Fernão Dias - Fernão Dias Environmentally Protected Area; EPA-SUL - Environmentally Protected Area of the Greater metropolitan area of Belo Horizonte-southern zone; Caratinga - Caratinga Biological Station; Duas Barras - Duas Barras Ranch; Ilha do Mel - Ilha do Mel; Jureia - Maciço da Juréia; Ibitipoca - Ibitipoca State Park; Itacolomi - Itacolomi State Park; Jacupiranga - Jacupiranga State Park; Rio Doce - Rio Doce State Park; Brigadeiro - Serra do Brigadeiro State Park; Intendente - Serra do Intendente State Park; Papagaio - Serra do Papagaio State Park; Chapada - Chapada Diamantina National Park; Itatiaia - Itatiaia National Park; Itajai - Serra de Itajaí National Park; A. Ruschi - Augusto Ruschi Biological Reserve; R.R. Pedras - Rio das Pedras Reserve; Caraça - Santuário do Caraça (privately owned) Nature Reserve; S. Bonita - Serra Bonita (privately owned) Nature Reserve; Cuscuzeiro - Serra do Cuscuzeiro; S. Mar-PR - Serra do Mar Paranaense; Serra Negra - Serra Negra.
Park has a strong floristic influence from the shrublands domain (Zappi 2008), which might explain the low correlation with other areas of the Espinhaço Range included in the analysis, which are influenced by the Atlantic Forest or cerrado domains. The Serra do Cuscuzeiro is an area with cerrado formations located in a region with a unique geological formation, the sandstone-basalt cuestas, which could be an explanation for the presence of a different assemblage of lycophytes and monilophytes and a low correlation with the other areas. Itatiaia National Park and Serra do Papagaio State Park are influenced by campos de altitude ("upland") grasslands and also show the highest altitudinal gradient among the areas, which provides a greater diversity of habitats and, hence, taxonomic diversity, including several endemic vascular plants (Brade 1956).

The similarity analysis corroborated the NMDS results and allowed areas of grasslands and forest formations to be differentiated from those composed exclusively of forest formations (Fig. 2). All of the areas located within the Minas Gerais domain of the Espinhaço Range showed floristic similarity (Fig. 2; Tab. 4). The following areas showed high Sørensen similarity index values in relation to the RPSP (Tab. 4): Serra do Intendente State Park, located in the southern part of the Espinhaço Range; Itacolomi State Park and the privately owned Santuário do Caraça Nature Reserve, both located in the Quadrilátero Ferrífero region; Ibitipoca and Serra Negra State Parks, in the southern part 
of Minas Gerais; and Chapada Diamantina National Park, in the northern part of the Espinhaço Range.

Considering the location of RPSP, a more prominent influence of cerrado on the floristic composition of the lycophyte and monilophyte flora could be expected. However, several species typical of the Atlantic Forest were found, reflecting the influence of the forest formations occurring above $1500 \mathrm{~m}$. However, as observed in the results of the analyses above, this influence appears to comes more from the inland rainforests than from the coastal ones. Such formations allow colonization by these taxa, as observed by Salino \& Almeida (2008a) in the Planalto de Diamantina region.

The results of our analyses show that RPSP, as well as other areas in the Espinhaço Range, presents a characteristic set of monilophyte and lycophyte species. Although the floristic composition is influenced by the Atlantic Forest, especially in the high mountain forests, the association of these forests with typical cerrado formations makes these environments conducive to a unique assemblage of species, although we can observe that the environmental characteristics present in the Espinhaço Range also occur in areas outside these mountains, such as in
Ibitipoca and Serra Negra State Parks. Almeida (2008) pointed out the high floristic similarity between these areas and suggested that, although within the Atlantic Forest and near the Mantiqueira Range, the Ibitipoca and Serra Negra State Parks also present grasslands, including rupestrian grasslands, as well as mata nebular formations that are similar to those of the Espinhaço Range. Obtaining greater clarification on the floristic relations between the communities of lycophytes and monilophytes occurring in different vegetation formations in Brazil will require more detailed analyses including all currently available data from floristic surveys.

Despite the recent increase in the sampling effort in the Planalto de Diamantina region, the results of the present study suggest that there is still a lack of knowledge about the local lycophyte and monilophyte flora, given that we recorded several species previously unknown to occur in the region. The increasing richness of lycophytes and monilophytes in the Planalto de Diamantina region, together with the occurrence of rare and threatened species in the area, underpins the initiative to expand the area of RPSP and underscores its importance in the conservation of this group of plants.

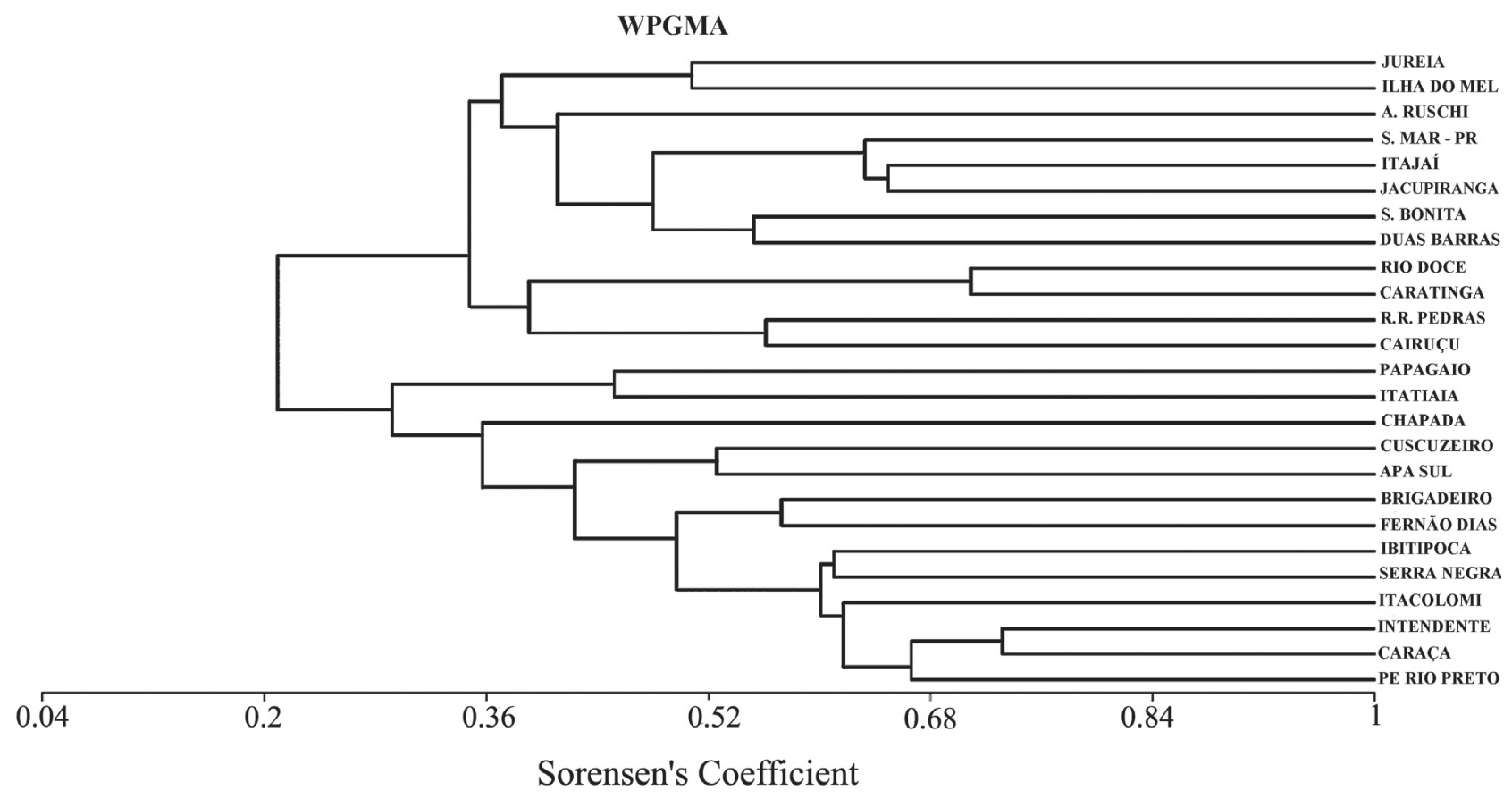

Figure 2. Dendrogram showing pteridophyte flora similarity among 25 areas included in the similarity analysis using the Sørensen similarity index and the weighted pair-group method with arithmetic mean (WPGMA) algorithm.

PE RIO PRETO - Rio Preto State Park; CAIRUÇU - Cairuçu Environmentally Protected Area; FERNÃO DIAS - Fernão Dias Environmentally Protected Area; EPA-SUL - Environmentally Protected Area of the Greater metropolitan area of Belo Horizonte-southern zone; CARATINGA - Caratinga Biological Station; DUAS BARRAS - Duas Barras Ranch; ILHA DO MEL - Ilha do Mel; JUREIA - Maciço da Juréia; IBITIPOCA - Ibitipoca State Park; ITACOLOMI - Itacolomi State Park; JACUPIRANGA - Jacupiranga State Park; RIO DOCE - Rio Doce State Park; BRIGADEIRO - Serra do Brigadeiro State Park; INTENDENTE - Serra do Intendente State Park; PAPAGAIO - Serra do Papagaio State Park; CHAPADA - Chapada Diamantina National Park; ITATIAIA - Itatiaia National Park; ITAJAI - Serra de Itajaí National Park; A. RUSCHI - Augusto Ruschi Biological Reserve; R.R. PEDRAS - Rio das Pedras Reserve; CARAÇA - Santuário do Caraça (privately owned) Nature Reserve; S. BONITA - Serra Bonita (privately owned) Nature Reserve; CUSCUZEIRO - Serra do Cuscuzeiro; S. MAR-PR - Serra do Mar Paranaense; SERRA NEGRA - Serra Negra. 


\section{Acknowledgments}

We thank the Instituto Estadual de Florestas de Minas Gerais (IEF-MG, Minas Gerais State Forestry Institute) for the logistical support and permits, as well as the entire staff of Rio Preto State Park for their support, especially the manager, Antonio Augusto Tonhão de Almeida, and the park employee known as Deco. We are also grateful to Dr. Pedro Lage Viana and Daniel Teixeira Souza for their assistance in the sample collection; Filipe de Souza Soares and Danilo Rafael Mesquita Neves for their help with the analyses; and Fernando Matos for his suggestions and revisions.

\section{References}

Abreu, A.A.D. 1984. O Planalto de Diamantina: um setor da Serra do Espinhaço em Minas Gerais. Orientação 5: 75-79.

Almeida, T.E. 2008. Análise quantitativa da distribuição geográfica das espécies de pteridófitas ocorrentes no estado de Minas Gerais, Brasil. Dissertação de Mestrado, Universidade Federal de Minas Gerais, Belo Horizonte.

Aquije, G.M.F.V. \& Santos, I.K.F. 2007. Levantamento Florístico de Pteridófitas da Reserva Biológica Augusto Ruschi, Santa Teresa, ES. Revista Brasileira de Biociências 5(2): 909-911.

Brade, A.C. 1956. A flora do Parque Nacional do Itatiaia. Boletim do Parque Nacional do Itatiaia 5: 7-85.

Condack, J.P.S. 2006. Pteridófitas ocorrentes na região alto Montana do Parque Nacional do Itatiaia: análise florística e estrutural. Dissertação de Mestrado, Jardim Botânico do Rio de Janeiro, Rio de Janeiro.

Drummond, G.M.; Martins, C.S.; Machado, A.B.M.; Sebaio, F.A. \& Antonini, Y. 2005. Biodiversidade em Minas Gerais: um atlas para sua conservação. Belo Horizonte, Fundação Biodiversitas.

Drummond, G.M; Machado, A.B.M.; Martins, C.S.; Mendonça, M.P. \& Stehmann, J.R. 2008. Listas vermelhas das espécies da fauna e da flora ameaçadas de extinção em Minas Gerais. 2 ed. Belo Horizonte, Fundação Biodiversitas.

Figueiredo, J.B. \& Salino, A. 2005. Pteridófitas de quatro Reservas Particulares do Patrimônio Natural ao Sul da Região Metropolitana de Belo Horizonte, Minas Gerais, Brasil. Lundiana 6(2): 83-94.

Gasper, A.L. \& Sevegnani, L. 2011. Lycophyta e samambaias do Parque Nacional da Serra do Itajaí, Vale do Itajaí, SC, Brasil. Hoehnea 37(4): 755-767.

Giulietti, A.M.; Menezes, N.L.; Pirani, J.R.; Meguro, M. \& Wanderley, M.G.L. 1987. Flora da Serra do Cipó, Minas Gerais: caracterização e lista das espécies. Boletim de Botânica da Universidade de São Paulo 9: 1-151.

Giulietti, A.M.; Pirani, J.R. \& Harley, R.M. 1997. Espinhaço Range region. Pp. 397-404. In: S.D. Davis, V.H. Heywood; O. Herrera-MacBryde; J. Villa-Lobos \& A. Hamilton (eds.). Centres of plant diversity: a guide and strategy for their conservation. Volume 3: The Americas. Cambridge, IUCN Publications Unit.

Gontijo, B.M. 2008. Uma geografia para a Cadeia do Espinhaço. Megadiversidade 4(1-2): 7-14.

Harley, R.M. 1995. Introduction. Pp. 1-42. In: B.L.E. Stannard (ed.). Flora of the Pico das Almas, Chapada Diamantina-Bahia, Brasil. Kew, Royal Botanical Garden.

IEF - Instituto Estadual de Florestas. 2004. Plano de Manejo do Parque Estadual do Rio Preto. Belo Horizonte, IEF.

Jacobi, C.M. \& Carmo, F.F. 2008. Diversidade dos campos rupestres ferruginosos no Quadrilátero Ferrífero, MG. Megadiversidade 4(1-2): 24-32.

Kent, M. \& Coker, P. 1992. Vegetation description and analysis. London, John Wiley \& Sons.

Köppen, W. 1931. Grundriss der Klimakunde. Berlin, Walter de Gruyter.

Legendre, P. \& Legendre, L. 1998. Numerical Ecology. Amsterdam, Elsevier.
Leite, F.S.F.; Juncá, F.A. \& Eterovick, P.C. 2008. Status do conhecimento, endemismo e conservação de anfíbios anuros da Cadeia do Espinhaço, Brasil. Megadiversidade 4(1-2): 182-200.

Magurran, A.E. 2004. Measuring Biological Diversity. Oxford, Blackwell Science.

Matos, F.B.; Amorim, A.M. \& Labiak, P.H. 2010. The ferns and lycophytes of a montane tropical forest in Southern Bahia, Brazil. Journal of the Botanical Research Institute of Texas 4(1): 333 - 346

MMA - Ministério do Meio Ambiente. 2008. Lista Oficial das Espécies Brasileiras Ameaçadas de Extinção. Instrução Normativa nº 6, 23 de setembro de 2008.

McCune, B. \& Mefford, M.J. 2006. PC-ORD. Multivariate analysis of ecological data, version 5.10. Gleneden Beach, Mjm Software.

Melo, L.C.N. \& Salino, A. 2002. Pteridófitas de duas áreas de floresta da Bacia do Rio Doce no Estado de Minas Gerais, Brasil. Lundiana. 3(2): 129-139.

Melo, L.C.N. \& Salino, A. 2007. Pteridófitas em fragmentos florestais da APA Fernão Dias, Minas Gerais, Brasil. Rodriguésia 58(1): 207-220.

Minchin, P. 1987. An evaluation of the relative robustness of techniques for ecological ordination. Plant Ecology 69: 89-107.

Moran, R.C. 2008. Diversity, biogeography, and floristics. Pp. 367-394. In: T.A. Ranker \& C.H. Haufler (eds.). Biology and Evolution of Ferns and Lycophytes. Cambridge, Cambridge University Press.

Mynssen, C.M. \& Windisch, P.G. 2004. Pteridófitas da Reserva de Rio das Pedras, Mangaratiba, RJ. Rodriguésia 55(85): 125-156.

Nonato, F.R. 2005. Pteridófitas. In: F.A. Juncá; L. Funch; W. Rocha. (orgs.). Biodiversidade e Conservação da Chapada Diamantina. Brasília, Ministério do Meio Ambiente.

Øllgaard, B. 1992. Neotropical Lycopodiaceae, an overview. Annals of the Missouri Botanical Garden 79(3): 687-717.

Øllgaard, B. 2012. Nomenclatural changes in Brazilian Lycopodiaceae. Rodriguésia 63(2): 471-482.

Paciência, M.L.B. 2008. Diversidade de Pteridófitas em gradientes de altitude na Mata Atlântica do Estado do Paraná. Tese de Doutorado, Universidade de São Paulo, São Paulo.

Page, C.N. 1979. The diversity of ferns: an ecological perspective. Pp. $10-$ 56. In: A.F. Dyer (ed.). The experimental biology of ferns. London, Academic Press.

Prado, J. \& Labiak, P.H. 2001. Pteridófitas. In: M.C.H. Mamede, I. Cordeiro \& L. Rossi (orgs.). Flora vascular da Serra da Juréia, Município de Iguape, São Paulo, Brasil. Boletim do Instituto de Botânica 15: 83-86.

Pichi-Sermolli, R.E.G. 1996. Authors of scientific names in Pteridophyta. Kew, Royal Botanical Gardens.

Ponce, M.; Mehltreter, K. \& de la Sota, E.R. 2002. Análisis biogeográfico de la diversidade pteridofítica en Argentina y Chile continental. Revista Chilena de Historia Natural 75: 703-717.

Pryer, K.M.; Schuettpelz, E.; Wolf, P.G.; Schneider, H.; Smith, A.R. \& Cranfill, R. 2004. Phylogeny and evolution of ferns (monilophytes) with a focus on the early leptosporangiate divergences. American Journal of Botany 91(10): 1582-1598.

Rapini, A.; Ribeiro, P.L.; Lambert, S. \& Pirani, J.R. 2008. A flora dos campos rupestres da Cadeia do Espinhaço. Megadiversidade 4(1-2): 15-23.

Ribeiro, J.F. \& Walter, B.M.T. 1998. Fitofisionomias do Bioma Cerrado. Pp.89-166. In.: S.M. Sano \& S.P. Almeida. Cerrado: Ambiente e Flora. Planaltina, Empresa Brasileira de Pesquisa Agropecuária.

Rolim, L.B. 2007. Pteridófitas do Parque Estadual do Itacolomi, Minas Gerais, Brasil. Dissertação de Mestrado, Universidade Federal de Minas Gerais, Belo Horizonte.

Rothfels, C.J.; Sundue, M.A.; Kuo, L.-Y.; Larsson, A.; Kato, M.; Schuettpelz, E. \& Pryer, K.M. 2012. A revised family-level classification for eupolypod II ferns (Polypodiidae: Polypodiales). Taxon 61(3): 515-533.

Salino, A. 1996. Levantamento das pteridófitas da Serra do Cuscuzeiro, Analândia, SP, Brasil. Revista Brasileira de Botânica 19(2):173-178.

Salino, A.; Silva, S.M.; Dittrich, V.A.O. \& Britez, R.M. 2005. Pp. 85-101. Flora Pteridofítica. In: M. C.M. Marques \& R.M. Britez. (orgs.). História Natural e Conservação da Ilha do Mel. Curitiba, Editora UFPR.

Salino, A. \& Almeida, T.E. 2008a. Diversidade e conservação das pteridófitas na Cadeia do Espinhaço, Brasil. Megadiversidade 4(1-2): 78-98.

Salino, A. \& Almeida, T.E. 2008b. Pteridófitas do Parque Estadual do Jacupiranga, SP, Brasil. Acta Botanica Brasilica 22(4): 983-991. 
Salino, A. \& Almeida, T.E. 2009. Pteridófitas. Pp. 331-352. In: G.M. Drummond; C.S. Martins; M.B. Greco \& F. Vieira. (orgs.). Biota Minas: diagnóstico do conhecimento sobre a biodiversidade no estado de Minas Gerais subsídio ao Programa Biota Minas. Belo Horizonte, Fundação Biodiversitas.

Silva, A.T. 1984. Pteridófitas. Pp.: 33-34. In: O. Fidalgo \& V.L. Bononi (eds.). Técnicas de coleta, preservação e herborização de material botânico. São Paulo, Instituto de Botânica.

Sokal, R.R. \& Michener, C.D. 1958. A statistical method for evaluating systematic relationship. University of Kansas Society Bulletim 38:1409-1438.

Smith, A.R; Pryer, K.M.; Schuettpelz, E.; Korall, P.; Schneider, H. \& Wolf, P.G. 2008. Fern classification. Pp. 417-467. In: T.A. Ranker \& C.H. Haufler (eds.). Biology and Evolution of Ferns and Lycophytes. Cambridge, Cambridge University Press.
Souza, F.S.; Salino, A.; Viana, P.L. \& Salimena, F.R.G. 2012. Pteridófitas da Serra Negra, Minas Gerais, Brasil. Acta Botânica Brasilica 26(2): 378-390

Sylvestre, L.S. 1997. Pteridophyta. Pp. 44-49. In: M.C.M., Marques; A.S.F Vaz \& R., Marquete. Mapeamento da cobertura vegetal e listagem das espécies ocorrentes na Área de Proteção Ambiental de Cairuçu, Município de Parati, Rio de Janeiro. Rio de Janeiro, Jardim Botânico do Rio de Janeiro/Ministério do Meio Ambiente.

Vasconcelos, M.F; Lopes, L.E.; Machado, C.G. \& Rodrigues, M. 2008. As aves dos campos rupestres da Cadeia do Espinhaço: diversidade, endemismo e conservação. Megadiversidade 4(1-2): 221-241.

Viveros, R.S. 2010. Pteridófitas da Serra do Caraça, Minas Gerais, Brasil. Dissertação de mestrado, Universidade Federal de Minas Gerais, Belo Horizonte.

Zappi, D. 2008. Fitofisionomia da Caatinga associada à Cadeia do Espinhaço. Megadiversidade 4(1-2): 33-37. 